\title{
Survey observations of large-scale molecular outflows associated with intermediate-mass protostar candidates in the $\mathrm{OMC}-2 / 3$ region
}

\author{
Satoko Takahashi ${ }^{1}$, Y. Shimajiri ${ }^{1}$, S. Takakuwa ${ }^{3}$, M. Saito ${ }^{3}$ \\ and R. Kawabe ${ }^{3}$ \\ ${ }^{1}$ Department of Astronomical Science, Graduate University for Advanced Studies, National \\ Astronomical Observatory of Japan, Osawa 2-21-1, Mitaka, Tokyo 181-8588 Japan \\ email:satoko.takahashi@nao.ac.jp \\ ${ }^{2}$ Department of Astronomy, School of Science, University of Tokyo, Bunkyo, Tokyo 113-0033, \\ Japan. \\ ${ }^{3}$ ALMA Project Office, National Astronomical Observatory of Japan, Osawa 2-21-1, Mitaka, \\ Tokyo 181-8588 Japan
}

\begin{abstract}
We have newly performed millimeter- and submillimeter-wave observations in the nearest GMC: the Orion Molecular Cloud $-2 / 3$ region (OMC-2/3). Here, we report results of our large-scale $\left(22^{\prime} \times 14^{\prime}\right)$ outflow survey with the Atacama Submillimeter Telescope Experiment (ASTE) in the $\mathrm{CO}(3-2)$ emission. The OMC-2/3 region is one of the famous intermediate-mass star-forming regions and harbors several sources diagnosed as Class0 protostars (Chini et al. 1997). With the intensive ASTE observations, we totally identified the 8 clear, 5 probable and 6 marginal outflows in OMC-2/3. 8 clear outflows from them, MMS 2, MMS 5, MMS7, MMS9, FIR-2, FIR 3, VLA 13, and FIR 6b are associated with $\mathrm{mm}$ and SPITZER $24 \mu \mathrm{m}$ sources. The others are more or less complicated, and two of which, VLA 13 and FIR 6, are newly identified. We found the interaction between the molecular outflows and the dust condensations at least in four regions. In addition, we confirmed the increment of the velocity width of the dense gas toward some of these condensations (i.e. at the termination of the outflow lobes). These results suggest that (i) the interaction between the outflows and the dense condensation occurs commonly in the OMC- $2 / 3$ region, (ii) the dense condensations in this region are compressed ubiquitously by these outflows and are receiving a part of the momentum from them. Particularly, one of the strongest millimeter sources, and hence protostar candidates, FIR4, is strongly compressed by a molecular outflow driven by FIR3 located at the north-east of FIR 4 . These results suggest that the molecular outflows play an important role in the formation and evolution of stars and that the outflows are a driving mechanism of turbulence in the OMC- $2 / 3$ region.
\end{abstract}

Keywords. molecular outflow, OMC-2/3. 